\title{
Aminoglycosides: Ancient and Modern
}

\author{
Julian E. Davies
}

\begin{abstract}
This short review is written as a tribute to Ken Rinehart, who was a real expert in aminoglycoside chemistry. A good friend and enthusiastic scientific collaborator, Ken would have contributed much to research in the current, developing field of aminoglycoside research.
\end{abstract}

Received: July 31, 2006 / Accepted: September 15, 2006

(C) Japan Antibiotics Research Association

\section{Keywords Rinehart}

\section{Discovery and Mode of Action}

The aminoglycosides were the first important therapeutic agents produced by bacterial fermentation. Streptomycin was discovered in Selman Waksman's laboratory in 1944 and neomycin in 1949; subsequently, kanamycin was reported by Hamao Umezawa in 1957, and gentamicin in 1963 by Marvin Weinstein's group at Schering Plough. In recent years many related compounds have been isolated by fermentation or by semisynthetic approaches. After 60 years of use the aminoglycosides still have an important role in the treatment of infectious diseases on a worldwide basis. The compounds are chemically stable and rapidly bactericidal, with a broad spectrum of inhibitory activity (apart from anaerobic bacteria).

All of the aminoglycosides have essentially the same mode of antibacterial action; they inhibit bacterial protein synthesis by binding to specific sites on the $30 \mathrm{~S}$ subunit of the $70 \mathrm{~S}$ prokaryotic ribosome. There has been significant progress in mode-of-action studies as a result of the determination by high resolution NMR analyses [1] and X-ray crystallographic structures [2] of various aminoglycosides bound to $16 \mathrm{~S}$ ribosomal RNA or the $30 \mathrm{~S}$ subunit. While the binding sites of different

J. E. Davies: Department of Microbiology and Immunology, Life Sciences Centre, University of British Columbia, Vancouver BC, V6T 1Z3, Canada, E-mail: jed@interchange.ubc.ca aminoglycoside classes differ in detail, they all bind to $16 \mathrm{~S}$ rRNA in a pocket of ribosomal proteins; these well-defined three-dimensional structures provide an explanation for their action in interfering specifically with transfer RNA interactions with messenger RNA in the decoding site of the ribosome. The results of this exciting new work satisfactorily describe the distinct molecular modes of action of the various classes of aminoglycosides and clearly delineate the differences between the binding of streptomycin containing streptamine and that of compounds containing 2-deoxystreptamine. The importance of the early work on aminoglycosides and their interactions with the ribosome was not appreciated at the time nor its significant role in what is now known as chemical biology. Studies with these antibiotics in the 1960s provided the first demonstrations that the ribosome has a vital role in the mechanism of reading of the genetic code for the determination of protein sequence.

\section{Applications}

It has been known for some time that certain aminoglycosides display activity against protein synthesis on the $80 \mathrm{~S}$ ribosomes of eukaryotes; the difference in binding specificity on both the $70 \mathrm{~S}$ ribosomes of prokaryotes and $80 \mathrm{~S}$ ribosomes of eukaryotes is due to an evolutionarily conserved base difference in the $16 / 18 \mathrm{~S}$ rRNA (A1408G). The activity of G-418 (geneticin) and hygromycin against eukaryotic cells has been widely exploited for the design of recombinant DNA cloning vectors for use in the genetic manipulation of yeasts, fungi, plants and mammalian cells; a cloned aminoglycoside- 
modifying enzyme (resistance) gene, usually an aminoglycoside phosphotransferase (APH) gene (aph $\left.3^{\prime}\right)$, provides the counter-selective marker.

The identification and characterization of rRNA as the essential ribosome component for the binding of the aminoglycosides has led to increased interest in the RNAbinding properties of this class of antibiotics, especially in the development of different types of therapeutic agents; creative approaches to antiviral therapy are one such outcome. The compounds have been demonstrated to bind specifically to a limited number of RNA sequences and shown to interfere with reactions such as the splicing of introns and certain ribozyme-catalysed functions. The therapeutic potential of RNA-binding molecules is considerable and the aminoglycosides promise to figure strongly in future studies [3]. It is also of interest to note that the early observation of the action of aminglycosides in causing codon mistranslation in bacteria (such as readingthrough stop codons), termed "phenotypic suppression" by Luigi Gorini, has been 'rediscovered', and a number of clinical studies have been carried out using aminoglycosides to suppress premature peptide-chain termination in genetic diseases such as thalassemias and cystic fibrosis [4]. Temporary alleviation of the symptoms of these serious afflictions using this treatment has been reported and studies are continuing.

\section{Resistance}

Despite their high potency, the aminoglycosides are used for antibacterial therapy less frequently than in the past. This is due in part to their renal and vestibular toxicity, but perhaps more importantly because of the incremental increase in the appearance and worldwide distribution of resistant bacterial strains exhibiting various cross-resistance patterns. Apart from mutations leading to alterations in antibiotic uptake by the cell or alteration of binding sites on the ribosome, there are probably more enzymatic mechanisms for aminoglycoside resistance than for any other class of antibiotic! Currently, some seven biochemically distinct groups of enzymes that modify different amino- or hydroxy-groups of the antibiotics are known, and within each class there are numerous allelic forms that encode discrete protein products; in total, more than fifty different modifying enzymes distributed among the seven enzymatic classes have been identified [5]. In addition, at least three 16S ribosomal RNA methylases that determine resistance by blocking antibiotic binding to the ribosome are known.

All these genetic determinants are acquired by horizontal gene transfer and the diversity of the clinically significant aminoglycoside resistance mechanisms found in hospitals mirrors the wide range of such genes found in the environment. This would suggest that the 'resistance' mechanisms are closely associated with the ecology and natural roles of these compounds, although their functions in nature remain unknown. However, based on amino acid sequence comparisons, the APH enzymes are closely related to protein kinases and the aminoglycoside acetyltransferase (AAC) enzymes are members of the GCN5-related $N$-acetyltransferase (GNAT) superfamily. Somewhat surprisingly, the gene for the widely distributed AAC (6')-Ib has evolved to modify an unrelated substrate, the fluoroquinolones, thus creating the first identified enzymic modification of these synthetic antimicrobials [6]. The new resistance gene is plasmid-encoded and can be horizontally transferred. One wonders what other functions may evolve from these broadly active gene classes.

\section{Transcription Modulation}

Apart from the well-established mode of action of the aminoglycosides as protein synthesis inhibitors, these antibiotics, as with all bacterial inhibitors, have significant, multifarious activities on cells. In the past five years our laboratory has demonstrated that extensive transcription responses are induced by various classes of antibiotic (including the aminoglycosides) at subinhibitory concentrations [7]. The evidence suggests that as a result both susceptible and resistant strains may display significant phenotypic alterations. It is reasonable to suppose that the known phenotypic variations induced by aminoglycosides may all be caused by transcription modulation. The analyses have revealed new aspects of the biology of aminoglycosides and other antibiotics and have identified a novel and unsuspected consequence of antibiotic binding to ribosomes.

Detection of alterations in cellular transcription patterns may provide a useful approach to the identification of novel biologically active compounds at subinhibitory concentrations; it is a simple and readily automated technique for screening the bioactivities contained in natural product mixtures. Different structural classes of aminoglycosides can be readily discriminated by reporter-based transcription profiling, despite the fact that they bind to closely related sites on the ribosome. Antibiotic "signatures" could be used in inhibitor identification and mode-of-action studies. Aminoglycoside resistance mutations have long been known to lead to some remarkable phenotypic changes; the first definitive studies were done in the laboratory of Luigi Gorini in the mid1960s [8]. More recently, Kozo Ochi and co-workers have carried out exciting work showing the enhanced production of various antibiotics in aminoglycoside-resistant mutants of Actinomycetes and Bacillus species [9]. 


\section{Future Prospects}

There has been relatively limited use of recombinant approaches to the manipulation of the structure of aminoglycosides as compared to the polyketide and nonribosomal peptide classes of antibiotics; the 'rules' of assembly in the latter cases are quite well known. To date, only a small number of aminoglycoside biosynthetic clusters have been cloned and their expression in heterologous hosts has been little studied. Apart from the studies of Terihiko Beppu, Suehara Horinouchi and Wolfgang Piepersberg on streptomycin production, there is very limited information on the genetics of aminoglycoside production and its regulation. The genome of only one aminoglycoside-producing strain, Streptomyces griseus, has been completely sequenced. A concerted effort in genomic and molecular genetic approaches would likely provide genetic methods for yield improvement and also for the production of novel aminoglycosides with improved activity against resistant strains. It is surprising that effective industrial processes for the microbial production of semisynthetic aminoglycosides, e.g. amikacin, have not been developed. Mutant-feeding studies (mutasynthesis) have met with limited success in the production of novel aminoglycosides (see below). With ample molecular genetic tools now available for the manipulation of actinomycetes and related antibiotic-producing bacteria, the time is ripe for the application of modern approaches in microbial genomics to the study of aminoglycosides!

\section{KLR and JED}

Ken Rinehart and I were consultants for the Upjohn Company in the early 1970s and we quickly established a good relationship and research collaboration. Tom Shier and Ken had published several papers on their creative mutasynthetic approach to the biotransformation of aminoglycosides employing mutants defective in the production of 2-deoxystreptamine (DOS). The basis of their work was the isolation of DOS-deficient $\left(\mathrm{D}^{-}\right)$strains of the neomycin producer Streptomyces fradiae, and subsequently other aminoglycoside-producing strains, following chemical mutagenesis $[10,11]$. The $\mathrm{D}^{-}$mutants were found to be dependent on the addition of DOS, or a close derivative, to enable the synthesis of an aminoglycoside. A wide variety of compounds were fed to the deficient mutants and a number provided active antibiotics. Tom and Ken called the products the hybrimycins. Since my laboratory was working on the mode of action of the aminoglycosides and their resistance mechanisms at the time, Ken gave us samples of the various hybrimycins, which we examined for their activity in cell-free protein synthesis systems from sensitive and resistant strains. The results provided some useful structure/activity relationships $[12,13]$. Other researchers have carried out similar types of mutant-feeding studies with other aminoglycosides; some years later, workers at Schering-Plough isolated $\mathrm{D}^{-}$mutants of Micromonospora inyouensis that were blocked in the production of sisomicin and employed medium supplementation with a variety

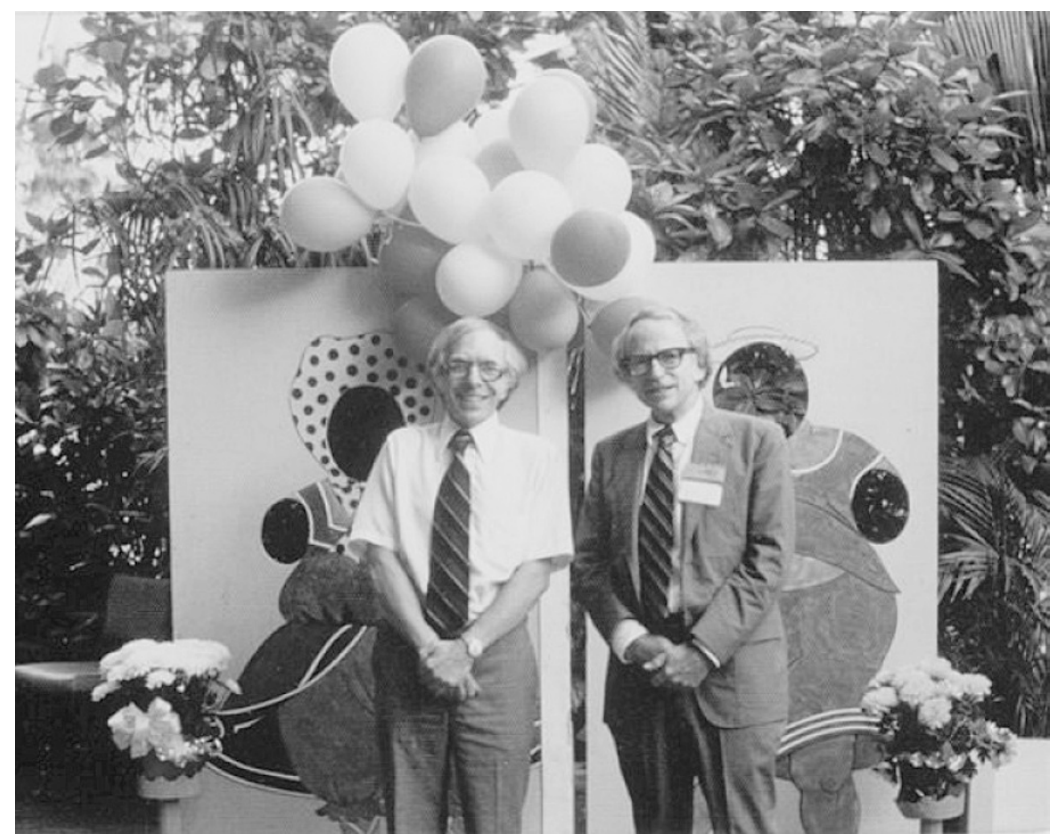

Fig. 1 
of DOS to produce analogues of this aminoglycoside. This work led to the identification of a compound, 5-episisomicin that had improved antibacterial activity compared to gentamicin and also inhibited certain classes of gentamicin-resistant bacteria; it eventually became the basis of a useful therapeutic agent [14]. We found that 5episisomicin has very good potency in blocking certain ribozyme-based reactions. It would be interesting to screen some of the other mutasynthetic derivatives of the aminoglycosides for such unsuspected biological activities.

Ken and I became good friends as a result of this collaboration, and he was always generous in providing me with a number of antibiotics and their derivatives, and much useful advice. We attended numerous meetings together and both were recipients of generous grants from the NIH Institute for Allergy and Infectious Diseases. Ken was a fine man and a great antibiotic chemist who made many important contributions in the applications of mass spectrometry to natural products. His interests extended beyond the aminoglycosides and he was a keen sportsman, being a diver, climber, and skier. We once skied together at Jackson Hole; Ken was much better than I! The deterioration in the past few years in the health of a man who was always so energetic in sport and agile of mind was tragic to see.

We shared some grant reviewing activity and our NIH Study Section Administrator claimed that Ken and I looked like twins. You can judge for yourself in the attached photograph taken at a meeting in Hawaii (Fig. 1); I am wearing a pair of Ken's spectacles. We last met in 1997 at a meeting at Rutgers University to celebrate the 50th anniversary of Selman Waksman's discovery of neomycin. Ken was obviously not in good health, but he gave, as usual, a highly knowledgeable account of the chemistry and biology of neomycin and related compounds. Ken Rinehart was a great natural product chemist who had a real appreciation for biology and I consider him to be one of the forerunners of what is now termed chemical biology. His passing is much regretted, but he left a lasting legacy.

Acknowledgements Thanks are due to the National Institutes of Health, the Canadian Institute for Health Research and the Canadian Bacterial Diseases Networks for many years of research support.

\section{References}

1. Ogle J, Ramakrishnan V. Structural insights into translational fidelity. Annu Rev Biochem 74: 129-177 (2005)

2. Lynch S, Gonzalez R, Puglisi J. Comparison of X-ray crystal structure of the $30 \mathrm{~S}$ subunit-antibiotic complex with NMR structure of decoding site oligonucleotide-paromomycin complex. Structure 11: 43-53 (2003)

3. Schroeder R, Waldsich C, Wank H. Modulation of RNA function by aminoglycoside antibiotics. Embo J 19: 1-9 (2000)

4. James P, Raut S, Rivard G, Poon M-C, McKenna S, Leggo J, Lillicrap D. Aminoglycoside suppression of nonsense mutations in severe hemophilia. Blood 106: 3043-3048 (2005)

5. Magnet S, Blanchard J. Molecular insights into aminoglycoside action and resistance. Chem Rev 105: 477-497 (2005)

6. Robicsek A, Strahilevitz J, Jacoby GA, Macielag M, Abbanat D, Park CH, Bush K, Hooper DC. Fluoroquinolone-modifying enzyme: a new adaptation of a common aminoglycoside acetyltransferase. Nat Med 12: 83-88 (2006)

7. Goh E, Yim G, Tsui W, McClure J, Surette M, Davies J. Transcriptional modulation of bacterial gene expression by subinhibitory concentrations of antibiotics. Proc Natl Acad Sci USA 99: 17025-17030 (2002)

8. Gorini L, Kataja E. Phenotypic repair by streptomycin of defective genotypes in E. coli. Proc Natl Acad Sci USA 51: 487-493 (1964)

9. Hosoya Y, Okamoto K, Murakami H, Ochi K. Acquisition of certain streptomycin-resistant (str) mutations enhances antibiotic production in bacteria. Antimicrob Agents Chemother 42: 2041-2047 (1998)

10. Shier W, Rinehart KL Jr, Gottlieb D. Preparation of four new antibiotics from a mutant of Streptomyces fradiae. Proc Natl Acad Sci USA 63: 198-204 (1969)

11. Shier WT, Rinehart KL Jr. Preparation of two new aminoglycoside antibiotics. J Antibiot 23: 51-53 (1970)

12. Davies J. Structure-activity relationships among the aminoglycoside antibiotics: comparison of the neomycins and hybrimycins. Biochim Biophys Acta 222: 674-676 (1970)

13. Benveniste R, Davies J. Structure-activity relationships among the aminoglycoside antibiotics: role of hydroxyl and amino groups. Antimicrob Agents Chemother 4: 402-409 (1973)

14. Waitz JA, Miller GH, Moss E, Jr, Chiu PJ. Chemotherapeutic evaluation of 5-episisomicin (Sch 22591), a new semisynthetic aminoglycoside. Antimicrob Agents Chemother 13: 41-48 (1978) 